\title{
New progress in the treatment of neuromyelitis optica spectrum disorder with monoclonal antibodies (Review)
}

\author{
QINFANG XIE, MENGJIAO SUN, JING SUN, TING ZHENG and MANXIA WANG \\ Department of Neurology, Lanzhou University Second Hospital, Lanzhou, Gansu 730030, P.R. China
}

Received July 29, 2020; Accepted November 27, 2020

DOI: $10.3892 /$ etm.2020.9579

\begin{abstract}
Neuromyelitis optica spectrum disorder (NMOSD) is a group of immune-mediated inflammatory demyelinating diseases mainly affecting the central nervous system. It is characterized by high risk of relapse and progression to disability. The frequent recurrences of neuromyelitis optica spectrum disorder often exacerbate the neurological dysfunction and severely affect the patient's quality of life. Conventional treatments for neuromyelitis optica spectrum disorder, including acute treatment and sequential therapy, aim to decrease the degree of disability and recurrences. In recent years, new monoclonal antibodies have yielded encouraging results. The present review discusses the research status and recent progress in the treatment of NMOSD with monoclonal antibodies.
\end{abstract}

\section{Contents}

1. Introduction

2. B cell depletion therapy

3. Interleukin (IL)-6 receptor (IL-6R) antagonists

4. Complement blockers

5. Other monoclonal antibodies

6. Summary

\section{Introduction}

Neuromyelitis optica spectrum disorder (NMOSD) is a group of inflammatory demyelinating diseases of the central nervous system that are mainly mediated by humoral immunity, and mainly involve the optic nerve and spinal cord (1). The aetiology of NMOSD is mainly associated with an aquaporin-4 antibody (AQP4-IgG), and $>80 \%$ of the patients are AQP4-IgG-positive (2). NMOSD usually occurs

Correspondence to: Dr Manxia Wang, Department of Neurology, Lanzhou University Second Hospital, 82 Cuiyingmen, Chengguan, Lanzhou, Gansu 730030, P.R. China

E-mail:wmx322@aliyun.com

Key words: neuromyelitis optica spectrum disorders, monoclonal antibodies, treatment in young adults and is more common in women compared with men. NMOSD has a high risk of relapse and progression to disability. Clinically, the treatment of NMOSD mainly involves glucocorticoids and immunosuppressive agents, mainly azathioprine (AZA), mycophenolate mofetil and cyclosporine. Over the years, as the understanding of NMOSD pathophysiology improved, many new therapies have emerged, including molecular-targeted therapy with monoclonal antibodies. The results of several recent phase III clinical trials on monoclonal antibodies have brought new hope for the treatment of NMOSD. This article reviews the latest progress in the treatment of NMOSD with monoclonal antibodies. The aim is to help researchers better understand the clinical application of monoclonal antibodies and to provide a reference for clinical practice.

\section{B cell depletion therapy}

B cells play a pathogenic role in NMOSD, an autoimmune disease of the central nervous system, through various mechanisms, including the production of pathogenic autoantibodies, secretion of cytokines/chemokines, and antigen presentation-T cell interactions (3). Therefore, the depletion of B cells by monoclonal antibodies has become a therapeutic strategy for NMOSD. Rituximab (RTX) and inebilizumab are the major monoclonal antibodies that are used in clinical practice to treat NMOSD by targeting B cells.

RTX. RTX is a human-mouse chimeric monoclonal antibody. It can effectively eliminate B cells produced by CD20-positive antibodies through complement- and antibody-dependent cytotoxicity and apoptosis. RTX was originally approved for the treatment of adult B-cell lymphoma. Later, the important role of B cells in some autoimmune diseases was discovered, so RTX has been gradually applied to the treatment of systemic lupus erythaematosus, rheumatoid arthritis, immune thrombocytopenic purpura, and myasthenia gravis (4,5). In 2005, Cree et al (6) first reported the experience of treating patients with neuromyelitis optica (NMO) using RTX. The results of this open study showed that six out of eight patients with NMO receiving RTX had no relapse during an average follow-up period of 12 months and that seven patients had significantly improved neurological function and a decrease in Expanded Disability Status Scale (EDSS) score from 7.5 before the treatment to 5.5. Several subsequent studies have demonstrated 
the high efficacy of RTX in the treatment of NMOSD (7-9). Therefore, RTX has been increasingly used in the treatment of NMOSD. The European Union of Neurology guideline in 2010 and the recommendations of the Neuromyelitis Optica Study Group in 2014 suggested the use of RTX as the first-line maintenance therapy for NMO $(10,11)$. However, these treatment experiences are mainly based on single-centre cohort studies, retrospective studies, or case reports. In 2016, Damato et al (12) published a systematic review on the efficacy and safety of RTX for NMOSD. The article included a total of 46 studies and conducted a meta-analysis on 25 of them. The results showed that RTX treatment effectively decreased the annual recurrence rate and EDSS score. A retrospective study by Poupart et al (13) found that RTX, as a first-line treatment for NMOSD, decreased the annual recurrence rate more effectively compared with mycophenolate mofetil but had no significant difference compared with AZA. The randomized controlled trial by Nikoo et al (14) showed that RTX was more effective than AZA in decreasing the annual recurrence rate and EDSS score in patients with NMOSD. In 2020, Tahara et al (15) published a study in The Lancet Neurology, which is the first randomized, double-blind, placebo-controlled clinical trial on the treatment of NMOSD with RTX. The study randomly divided the 38 patients with NMOSD into one group receiving $\operatorname{RTX}(n=19)$ and one group receiving placebo $(n=19)$. All patients received oral administration of low-dose hormones. During the 72-week follow-up period, relapse occurred in 7 (37\%) patients receiving placebo, and no relapse occurred in patients receiving RTX. In addition, the changes in the total quantification of nerve and spinal cord impairment (QOSI) scores were significantly higher in patients receiving RTX compared with those receiving placebo ( -1.16 vs. $0.63 ; \mathrm{P}=0.033)$.

Due to the lack of data, the use of B cell depletion therapy to treat NMOSD in pregnant patients has been controversial. Kim et al (16) retrospectively analyzed 29 pregnant patients with NMOSD and found that the pregnancy-associated relapse was negatively correlated with pre-pregnancy RTX treatment (odds ratio, 0.048; 95\% CI, 0.004-0.546). It was thus speculated that pre-pregnancy RTX treatment may help prevent pregnancy-associated NMOSD attack.

A five-year study indicated that RTX has good safety (17). The randomized controlled trial by Tahara et al (15) also showed that except for infusion reactions, the incidences of adverse events were similar between patients receiving RTX and placebo. Although RTX has been on the market for nearly two decades, the risk of progressive multifocal leukopathy, hypogammaglobulinemia, and severe infections cannot be neglected in patients who take RTX for a long time $(18,19)$. Rituximab has been included in treatment guidelines but has not been approved by United States Food and Drug Administration (FDA) for NMOSD (20).

Inebilizumab (MEDI-551). Inebilizumab is an anti-CD19 humanized monoclonal antibody that targets B cells and specifically binds to the CD19 antigen on the B cell surface, resulting in B cell depletion (21). CD19 antigen is a type I transmembrane glycoprotein belonging to the immunoglobulin (Ig) superfamily. It is expressed in early pro-B cells, late pro-B cells, memory B cells, plasmablasts, and some plasma cells (22). Compared with the anti-CD20 monoclonal antibody, the anti-CD19 antibody not only has better affinity but can also identify more B cell lineages. In recent years, inebilizumab has been tested in phase I clinical trials of systemic sclerosis and multiple sclerosis $(23,24)$. However, before the N-MOmentum study, inebilizumab had never been used in patients with NMOSD, nor has any published study supported its use in the treatment of NMOSD (25). The N-MOmentum study was a double-blind, randomized, placebo-controlled phase II/III clinical trial evaluating the efficacy of inebilizumab for the treatment of NMOSD (26). Two hundred thirty patients with NMOSD were randomly divided into a group receiving inebilizumab $(n=174)$ and a group receiving placebo $(n=56)$ at a ratio of 3:1. Patients received intravenous injections of $300 \mathrm{mg}$ inebilizumab or placebo on the 1st and 15 th days of the trial. The primary endpoint was the time to onset of an NMOSD attack. The study found that compared with placebo treatment, inebilizumab treatment significantly delayed the time to onset of an NMOSD attack. Twenty-one (12\%) of the 174 participants receiving inebilizumab and $22(39 \%)$ out of the 56 participants receiving placebo had an NMOSD attack. In addition, the risk of disability, the occurrence of new MRI lesions, and the number of hospital visits associated with NMOSD were significantly lower in participants receiving inebilizumab compared with those receiving placebo. In terms of safety, the N-MOmentum study notes that the common adverse reactions in the participants receiving inebilizumab included urinary tract infection, joint pain, back pain, headache, cystitis and sore eyes. In the non-blinded stage of the study, two patients died, one due to respiratory insufficiency caused by disease progression, the other due to inflammatory diseases of the central nervous system with unknown aetiology, and an association between death and treatment with inebilizumab could not be ruled out. In the future, long-term follow-up studies with a large sample are needed to further understand the safety of inebilizumab. In June 2020, inebiluzumab was approved by FDA for AQP4-IgG NMOSD (27).

\section{Interleukin (IL)-6 receptor (IL-6R) antagonists}

IL-6 can be elevated in the serum and cerebrospinal fluid of patients with NMOSD (28). Chihara et al (29) found that the increased expression of IL-6 in patients with NMO enhanced the viability of plasmablasts, thereby stimulating the secretion of AQP4-Ab. In vitro experiments confirmed that the blocking of IL-6R signalling by anti-IL-6R antibody can decrease the survival of plasmablasts. These results suggest that the IL-6-dependent B cell subsets are involved in the pathogenic mechanism of NMO, and IL-6R signalling may become a new target for NMO treatment. IL-6 may have multiple roles in NMOSD pathophysiology by stimulating antibody production in B cells and the development of effector T cells, by regulating the balance between Th17 and Treg cells, and by disruption of BBB function (30). The treatment of neuropathic pain is a difficult problem for patients with NMOSD. Increasing evidence indicates that IL-6R antagonists can not only effectively reduce the annual recurrence rate and EDSS scores but also alleviate neuropathic pain and fatigue symptoms in patients with NMOSD $(31,32)$.

Tocilizumab (TCZ). TCZ is a humanized anti-IL-6R monoclonal antibody of the IgG1 subclass and is the first anti-IL-6R 
monoclonal antibody used to treat NMOSD. Currently, this drug has been approved for the treatment of adult rheumatoid arthritis and juvenile rheumatoid arthritis (33). Araki et al (34) published the first study on TCZ to treat NMOSD in 2012. Only patient, a 36-year-old female with NMO, was studied, who received an intravenous injection of $8 \mathrm{mg} / \mathrm{kg}$ TCZ once a month for six months. After treatment, the number of relapses significantly decreased, the EDSS score decreased from 3.5 to 2.0 , and the pain numerical rating scale score decreased from 4 to 0 . Another study showed that TCZ significantly lowered the annual recurrence rate in three patients with NMO who responded poorly to RTX (35). Subsequently, Araki et al (32) published a study with a larger sample size. Seven patients with NMOSD were administered monthly injections of TCZ $(8 \mathrm{mg} / \mathrm{kg})$ in addition to original treatment. Following 12 months of treatment, the annual recurrence rate decreased from $2.9 \pm 1.1$ to $0.4 \pm 0.8$, and the EDSS score, neuropathic pain, and fatigue symptoms were all significantly improved. A retrospective study evaluating the long-term efficacy and safety of TCZ indicated that it is safe and effective to start TCZ as soon as possible for patients with refractory, highly active NMOSD, and that they should adhere to the monthly treatment plan (8 mg/kg) for a long time (36). Lotan et al (37) found that, like intravenous injection therapy, subcutaneous injection of TCZ decreased the annual recurrence rate of NMOSD ( 2 vs. $0 ; \mathrm{P}=0.0015$ ). The results indicated that subcutaneous injection might be a more convenient route for administration of TCZ in patients with NMOSD. In a recently published phase II randomized controlled clinical trial (TANGO trial), 118 patients with NMOSD were randomly divided into a group receiving TCZ $(n=59)$ and a group receiving AZA $(n=59)$ (38). In addition to the original treatment with immunosuppressive agents, TCZ or AZA was taken for a total of 60 weeks, and the results showed that the median time to first relapse was longer in patients taking TCZ compared with patients taking AZA (78.9 vs. 56.7 weeks; $\mathrm{P}=0.0026)$. At the end of the study, relapse occurred in $8(14 \%)$ patients taking TCZ and $28(47 \%)$ patients taking AZA (HR: 0.236; $\mathrm{P}<0.0001)$. The incidence of adverse reactions was similar in the two groups of patients, but the incidence of severe adverse reactions was higher in patients taking AZA compared with those taking TCZ. The results indicated that compared with AZA, TCZ had significantly lower relapse risk and better safety. Infection, leukopenia, anaemia and hypercholesterolaemia are the most common adverse reactions to TCZ in patients with NMOSD (39). During TCZ treatment, the complete blood count, liver and kidney function and blood lipids should be closely monitored to prevent thrombosis and cardiovascular events. TCZ has not been considered by FDA for NMOSD.

Satralizumab (SA237). SA237 is a novel anti-IL-6R monoclonal antibody. Compared with other anti-IL-6R monoclonal antibodies, SA237 has a longer half-life and can be administered by subcutaneous injection. Two recent phase III clinical trials, SAkuraSky and SAkuraStar, respectively described the efficacy of SA237 in combination drug therapy or monotherapy for NMOSD. SAkuraSky was a randomized, double-blind, placebo-controlled phase III clinical trial conducted by Yamamura et al (40) on the treatment of NMOSD with the combined use of SA237 and other immunosuppressive agents.
The trial was published in 2019 in the New England Journal of Medicine. In the trial, 83 patients with NMOSD were randomly allocated to receive SA237 $(n=41)$ or placebo $(n=42)$. On top of immunosuppressants, the patients received subcutaneous injections of $120 \mathrm{mg}$ of SA237 or placebo at weeks 0,2 and 4 and every 4 weeks thereafter. Relapse occurred in $8(20 \%)$ patients receiving SA237 and 18 (43\%) patients receiving placebo. The recurrence rates of patients receiving SA237 and patients receiving placebo were 0.11 and 0.32 , respectively. Subgroup analysis showed that among AQP4-IgG-seropositive patients, relapse occurred in 3/27 (11\%) patients who received SA237 and in 12/28 (43\%) patients who received placebo. Among AQP4-IgG-seronegative patients, relapse occurred in $5 / 14(36 \%)$ patients who received SA237, and in $6 / 14$ (43\%) patients who received placebo. The visual analogue scale scores and the Functional Assessment of Chronic Illness Therapy-Fatigue (ACIT-F) at 24 weeks were not significantly different between patients receiving SA237 and patients receiving placebo. Therefore, Yamamura et al (40) concluded that adding SA237 therapy to immunosuppressant therapy can decrease relapse in patients with NMOSD, especially AQP4-IgG-seropositive patients, and that SA237 is not different from placebo in its relief of pain or fatigue. The SAkuraStar study is a recently published randomized controlled trial on SA237 as monotherapy for patients with NMOSD (41). This trial included 95 patients with NMOSD, 63 of whom received SA237 and 32 placebo. The results showed that relapse occurred in $19(30 \% ; n=63)$ patients receiving SA237 and in $16(50 \%$; $n=32)$ patients receiving placebo. Similar to the SAkuraSky study, the changes in visual analogue scale pain score and FACIT-F fatigue score were not significantly different between SA237 and placebo groups. Subgroup analysis showed that among AQP4-IgG-seropositive patients, relapse occurred in $9(22 \%$; $n=41)$ patients receiving SA237 and in $13(57 \% ; n=23)$ patients receiving placebo and that among AQP4-IgG-seronegative patients, relapse occurred in $10(46 \% ; n=22)$ patients receiving SA237 and in $3(33 \%$; $\mathrm{n}=9$ ) patients receiving placebo. In summary, SAkuraSky and SAkuraStar indicated that monotherapy with SA237 can effectively decrease the recurrence rate and delay relapse in patients with NMOSD, especially AQP4-IgG-seropositive patients. The safety results of the two trials were similar. They both concluded that SA237 had good safety and that the most common clinical adverse reactions to SA237 were infection and injection-associated reactions, which were mostly mild to moderate. There were no deaths or anaphylactic reactions in either trials. A systematic review showed that the anti-IL-6 drugs TCZ and SA237 had good efficacy and safety in NMOSD (42). Nevertheless, a long course of treatment and long-term monitoring are still needed to assess the real risks of infection, neutropenia and cardiovascular side effects associated with these drugs (43). In August 2020, Satralizumab was approved for the treatment of NMOSD in adult patients who are AQP4 antibody-positive by FDA (44).

\section{Complement blockers}

Eculizumab. Eculizumab is a humanized monoclonal antibody that specifically binds to the complement protein $\mathrm{C} 5$ with high affinity, inhibits its cleavage to C5a and C5b, and 
prevents the formation of the terminal complement complex C5b-9, thereby inhibiting the activation of the terminal complement pathway (45). Eculizumab is mainly used for the treatment of paroxysmal nocturnal haemoglobinuria, atypical haemolytic uraemic syndrome and generalized myasthenia gravis. An open study in 2013 proposed that eculizumab may prevent the relapse of NMOSD (46). This study included 14 AQP4-IgG-seropositive female patients who received intravenous injections of $600 \mathrm{mg}$ eculizumab weekly for 4 weeks. At week 5 , the patients started to receive intravenous injections of $900 \mathrm{mg}$ eculizumab every 2 weeks. The treatment course lasted a total of 48 weeks. After 48 weeks of treatment, relapse only occurred in two out of 14 patients, and the EDSS score decreased from 4.3 before the treatment to 3.5 after the treatment $(\mathrm{P}=0.0078)$. In addition, five patients had improved visual acuity. The study concluded that eculizumab could significantly decrease the frequency of attack and maintain or improve neurological dysfunction in patients with NMOSD. However, the aforementioned studies were uncontrolled and unblinded clinical trials with small samples. In 2019, Pittock et al (47) published a randomized, double-blind clinical trial on eculizumab treatment in 143 AQP4-IgG-seropositive patients with NMOSD. The patients were randomly allocated to receive eculizumab $(n=96)$ or placebo $(n=47)$ in a ratio of $2: 1$. Each patient received $900 \mathrm{mg}$ of eculizumab or placebo once a week in the first 4 weeks. At week 5 , the patients started to receive intravenous injections of $1,200 \mathrm{mg}$ eculizumab every 2 weeks. Relapse occurred in 3 patients $(3 \% ; n=96$; annual recurrence rate, 0.02$)$ receiving eculizumab and in 20 patients (43\%; $n=47$; annual recurrence rate, 0.35 ) receiving placebo. However, there was no significant difference in the EDSS score between groups. Therefore, Pittock et al (47) concluded that the risk of relapse is significantly lower in AQP4-IgG-positive patients with NMOSD receiving eculizumab compared with those receiving placebo. The long-term efficacy of eculizumab in patients with NMOSD still requires further study. In terms of safety, since the C5b-9 complex has a bactericidal effect on encapsulated bacteria (especially Neisseria meningitidis), the blocking of the synthesis of the C5b-9 complex by eculizumab may increase the susceptibility of patients to infection. Therefore, in both studies, a meningococcal vaccine was given to the subjects before the start of the trial $(46,47)$. In the first study, one patient developed meningococcaemia and aseptic meningitis (46). In the second study, no meningococcal infection occurred (47). In addition, the most common adverse reactions reported in these two studies included upper respiratory tract infection, headache, nausea and dizziness. Currently, in the European Union, the United States, Canada and Japan, eculizumab has been approved for the treatment of AQP4-IgG-positive adult patients with NMOSD (48).

\section{Other monoclonal antibodies}

Several other monoclonal antibodies are at the testing stage in animal experimental models or at the early clinical stage, which will be briefly introduced in the present review.

Aquaporumab (mAb-53) is a humanized non-pathogenic recombinant monoclonal antibody targeting AQP4. Like the pathogenic AQP4 antibody, the Fab fragment of aquaporumab competitively binds to the AQP4 protein, but the Fc fragment of aquaporumab is artificially mutated to eliminate the activation of complement- and cell-mediated cytotoxicity (49). Aquaporumab has not been applied to human patients with NMOSD. However, in vitro, aquaporumab in the culture medium effectively inhibits the binding of the AQP4 antibody to target cells expressing AQP4. In an in vitro spinal cord section model and a mouse model of $\mathrm{NMO}$, aquaporumab prevented the development of NMO injury and inhibited cytotoxicity (50). Duan et al (51) found that high-affinity aquaporumab formed by saturation mutagenesis prevented serum-induced cell injury in patients with NMO. The competitive blocking of AQP4-IgG binding and inhibition of cytotoxicity by aquaporumab may provide a new treatment strategy for AQP4-IgG-seropositive patients with NMOSD.

Bevacizumab is a recombinant humanized monoclonal antibody that targets the vascular endothelial growth factor. It can inhibit angiogenesis and maintain the integrity of the blood-brain barrier. It has been approved by the US Food and Drug Administration for use in a variety of malignant tumours. The serum in patients with NMO has excess vascular endothelial growth factor that destroys the blood-brain barrier (52). Mealy et al (53) conducted a phase Ib clinical trial on the treatment of NMOSD with bevacizumab. The trial included 10 patients. The EDSS scores of five patients in the follow-up period were better compared with those at the time of admission. In terms of safety, according to the previous experience of bevacizumab in the treatment of other diseases, the most serious adverse reactions to bevacizumab are haemorrhage and thromboembolism (54). However, in the trial conducted by Mealy et al (53), none of the 10 patients had these adverse reactions, and only one patient had serious infection (urinary tract infection), which was considered unrelated to the medication. Other adverse reactions included oedema, diarrhoea, rash and headache.

Ublituximab is a novel anti-CD20 monoclonal antibody that targets the specific epitope of the CD20 antigen on mature B lymphocytes. In a phase I open clinical trial, five AQP4-IgG-seropositive patients with NMOSD received ublituximab treatment in addition to intravenous infusion of methylprednisolone. In the 90-day follow-up period, the EDSS score of three patients improved, and the B cell depletion status of four patients lasted $>2$ months. This study also showed that ublituximab had good safety. All five patients had no serious infections, infusion reactions, or liver disease and the adverse reactions of patients mainly included transient leukopenia, severe headache, and body pain (55).

\section{Summary}

In summary, most of the aforementioned monoclonal antibodies are very effective and well tolerated in patients with NMOSD, particularly those with poor response to conventional immunosuppressive agents. The monoclonal antibodies targeting AQP4, such as aquaporumab, have higher disease specificity and thus may have better clinical effects and fewer adverse reactions. However, new monoclonal antibodies such as aquaporumab and ublituximab still require prospective, randomized, controlled studies with large samples to clarify their efficacy. 


\section{Acknowledgements}

Not applicable.

\section{Funding}

This work was supported by the Lanzhou Talent Innovation and Entrepreneurship Project (grant no. 2018-RC-74) and Lanzhou Science and Technology project (grant no. 2018-3-50).

\section{Availability of data and material}

Not applicable.

\section{Authors' contributions}

MW conceived the study and edited the manuscript. QX contributed to writing the manuscript. MS revised the manuscript. JS and TZ searched literature and polished the language. All authors read and approved the final manuscript.

\section{Ethics approval and consent to participate}

Not applicable.

\section{Patient consent for publication}

Not applicable.

\section{Competing interests}

The authors declare that they have no competing interests.

\section{References}

1. Wingerchuk DM, Banwell B, Bennett JL, Cabre P, Carroll W, Chitnis T, de Seze J, Fujihara K, Greenberg B, Jacob A, et al: International Panel for NMO Diagnosis. International consensus diagnostic criteria for neuromyelitis optica spectrum disorders Neurology 85: 177-189, 2015.

2. Waters P, Reindl M, Saiz A, Schanda K, Tuller F, Kral V, Nytrova P, Sobek O, Nielsen HH, Barington T, et al: Multicentre comparison of a diagnostic assay: Aquaporin-4 antibodies in neuromyelitis optica. J Neurol Neurosurg Psychiatry 87: 1005-1015, 2016.

3. Forsthuber TG, Cimbora DM, Ratchford JN, Katz E and Stüve O B cell-based therapies in CNS autoimmunity: Differentiating CD19 and CD20 as therapeutic targets. Ther Adv Neurol Disord 11: 1756286418761697, 2018.

4. Rastetter W, Molina A and White CA: Rituximab: Expanding role in therapy for lymphomas and autoimmune diseases. Annu Rev Med 55: 477-503, 2004.

5. Iorio R, Damato V, Alboini PE and Evoli A: Efficacy and safety of rituximab for myasthenia gravis: A systematic review and meta-analysis. J Neurol 262: 1115-1119, 2015.

6. Cree BA, Lamb S, Morgan K, Chen A, Waubant E and Genain C: An open label study of the effects of rituximab in neuromyelitis optica. Neurology 64: 1270-1272, 2005.

7. Jacob A, Weinshenker BG, Violich I, McLinskey N, Krupp L, Fox RJ, Wingerchuk DM, Boggild M, Constantinescu CS, Miller A, et al: Treatment of neuromyelitis optica with rituximab: Retrospective analysis of 25 patients. Arch Neurol 65: 1443-1448, 2008.

8. Bedi GS, Brown AD, Delgado SR, Usmani N, Lam BL and Sheremata WA: Impact of rituximab on relapse rate and disability in neuromyelitis optica. Mult Scler 17: 1225-1230, 2011.

9. Pellkofer HL, Krumbholz M, Berthele A, Hemmer B, Gerdes LA Havla J, Bittner R, Canis M, Meinl E, Hohlfeld R and Kuempfel T: Long-term follow-up of patients with neuromyelitis optica after repeated therapy with rituximab. Neurology 76: 1310-1315, 2011.
10. Sellner J, Boggild M, Clanet M, Hintzen RQ, Illes $Z$, Montalban X, Du Pasquier RA, Polman CH, Sorensen PS and Hemmer B: EFNS guidelines on diagnosis and management of neuromyelitis optica. Eur J Neurol 17: 1019-1032, 2010.

11. Trebst C, Jarius S, Berthele A, Paul F, Schippling S, Wildemann B, Borisow N, Kleiter I, Aktas O and Kümpfel T; Neuromyelitis Optica Study Group (NEMOS): Update on the diagnosis and treatment of neuromyelitis optica: Recommendations of the Neuromyelitis Optica Study Group (NEMOS). J Neurol 261: 1-16, 2014.

12. Damato V, Evoli A and Iorio R: Efficacy and safety of rituximab therapy in neuromyelitis optica spectrum disorders: A systematic review and meta-analysis. JAMA Neurol 73: 1342-1348, 2016.

13. Poupart J, Giovannelli J, Deschamps R, Audoin B, Ciron J, Maillart E, Papeix C, Collongues N, Bourre B, Cohen M, et al: Evaluation of efficacy and tolerability of first-line therapies in NMOSD. Neurology 94: e1645-e1656, 2020.

14. Nikoo Z, Badihian S, Shaygannejad V, Asgari N and Ashtari F: Comparison of the efficacy of azathioprine and rituximab in neuromyelitis optica spectrum disorder: A randomized clinical trial. J Neurol 264: 2003-2009, 2017.

15. Tahara M, Oeda T, Okada K, Kiriyama T, Ochi K, Maruyama H, Fukaura H, Nomura K, Shimizu Y, Mori M, et al: Safety and efficacy of rituximab in neuromyelitis optica spectrum disorders (RIN-1 study): A multicentre, randomised, double-blind, placebo-controlled trial. Lancet Neurol 19: 298-306, 2020.

16. Kim SH, Huh SY, Jang H, Park NY, Kim Y, Jung JY, Lee MY, Hyun JW and Kim HJ: Outcome of pregnancies after onset of the neuromyelitis optica spectrum disorder. Eur J Neurol 27: 1546-1555, 2020

17. Kim SH, Huh SY, Lee SJ, Joung A and Kim HJ: A 5-year follow-up of rituximab treatment in patients with neuromyelitis optica spectrum disorder. JAMA Neurol 70: 1110-1117, 2013.

18. Marcinnò A, Marnetto F, Valentino $\mathrm{P}$, Martire S, Balbo A, Drago A, Leto M, Capobianco M, Panzica G and Bertolotto A: Rituximab-induced hypogammaglobulinemia in patients with neuromyelitis optica spectrum disorders. Neurol Neuroimmunol Neuroinflamm 5: e498, 2018.

19. Luna G, Alping P, Burman J, Fink K, Fogdell-Hahn A, Gunnarsson M, Hillert J, Langer-Gould A, Lycke J, Nilsson P, et al: Infection risks among patients with multiple sclerosis treated with fingolimod, natalizumab, rituximab, and injectable therapies. JAMA Neurol 77: 84-191, 2020.

20. Holmøy T, Høglund RA, Illes Z, Myhr KM and Torkildsen $\varnothing$ : Recent progress in maintenance treatment of neuromyelitis optica spectrum disorder. J Neurol: Oct 3, 2020 (Online ahead of print).

21. Chen D, Gallagher S, Monson NL, Herbst R and Wang Y: Inebilizumab, a B cell-depleting anti-CD19 antibody for the treatment of autoimmune neurological diseases: Insights from preclinical studies. J Clin Med 5: 107, 2016.

22. Otero DC, Anzelon AN and Rickert RC: CD19 function in early and late B cell development: I. Maintenance of follicular and marginal zone B cells requires CD19-dependent survival signals. J Immunol 170: 73-83, 2003.

23. Schiopu E, Chatterjee S, Hsu V, Flor A, Cimbora D, Patra K, Yao W, Li J, Streicher K, McKeever K, et al: Safety and tolerability of an anti-CD19 monoclonal antibody, MEDI-551, in subjects with systemic sclerosis: A phase I, randomized, placebo-controlled, escalating single-dose study. Arthritis Res Ther 18: 131, 2016.

24. Agius MA, Klodowska-Duda G, Maciejowski M, Potemkowski A, Li J, Patra K, Wesley J, Madani S, Barron G, Katz E and Flor A: Safety and tolerability of inebilizumab (MEDI-551), an anti-CD19 monoclonal antibody, in patients with relapsing forms of multiple sclerosis: Results from a phase 1 randomised, placebo-controlled, escalating intravenous and subcutaneous dose study. Mult Scler 25: 235-245, 2019.

25. Paul F, Murphy O, Pardo S and Levy M: Investigational drugs in development to prevent neuromyelitis optica relapses. Expert Opin Investig Drugs 27: 265-271, 2018.

26. Cree BAC, Bennett JL, Kim HJ, Weinshenker BG, Pittock SJ, Wingerchuk DM, Fujihara K,Paul F, Cutter GR, Marignier R, et al: Inebilizumab for the treatment of neuromyelitis optica spectrum disorder (N-MOmentum): A double-blind, randomised placebo-controlled phase 2/3 trial. Lancet 394: 1352-1363, 2019.

27. Frampton JE: Inebilizumab: First approval. Drugs 80: 1259-1264, 2020.

28. Uzawa A, Mori M, Arai K, Sato Y, Hayakawa S, Masuda S, Taniguchi J and Kuwabara S: Cytokine and chemokine profiles in neuromyelitis optica: Significance of interleukin-6. Mult Scler 16: 1443-1452, 2010. 
29. Chihara N, Aranami T, Sato W, Miyazaki Y, Miyake S, Okamoto T, Ogawa M, Toda T and Yamamura T: Interleukin 6 signaling promotes anti-aquaporin 4 autoantibody production from plasmablasts in neuromyelitis optica. Proc Natl Acad Sci USA 108: 3701-3706, 2011.

30. Fujihara K, Bennett JL, de Seze J, Haramura M, Kleiter I, Weinshenker BG, Kang D, Mughal T and Yamamura T: Interleukin-6 in neuromyelitis optica spectrum disorder pathophysiology. Neurol Neuroimmunol Neuroinflamm 7: e841, 2020.

31. Bradl M, Kanamori Y, Nakashima I, Misu T, Fujihara K, Lassmann $\mathrm{H}$ and Sandkühler J: Pain in neuromyelitis optica - prevalence, pathogenesis and therapy. Nat Rev Neurol 10: 529-536, 2014

32. Araki M, Matsuoka T, Miyamoto K, Kusunoki S, Okamoto T, Murata M, Miyake S, Aranami T and Yamamura T: Efficacy of the anti-IL-6 receptor antibody tocilizumab in neuromyelitis optica: A pilot study. Neurology 82: 1302-1306, 2014.

33. Kang S, Tanaka T, Narazaki $M$ and Kishimoto T: Targeting interleukin-6 signaling in clinic. Immunity 50: 1007-1023, 2019.

34. Araki M, Aranami T, Matsuoka T, Nakamura M, Miyake S and Yamamura T: Clinical improvement in a patient with neuromyelitis optica following therapy with the anti-IL-6 receptor monoclonal antibody tocilizumab. Mod Rheumatol 23: 827-831, 2013.

35. Ayzenberg I, Kleiter I, Schröder A, Hellwig K, Chan A, Yamamura T and Gold R: Interleukin 6 receptor blockade in patients with neuromyelitis optica nonresponsive to anti-CD20 therapy. JAMA Neurol 70: 394-397, 2013.

36. Ringelstein M, Ayzenberg I, Harmel J, Lauenstein AS, Lensch E, Stögbauer F, Hellwig K, Ellrichmann G, Stettner M, Chan A, et al: Long-term therapy with interleukin 6 receptor blockade in highly active neuromyelitis optica spectrum disorder. JAMA Neurol 72 756-763, 2015.

37. Lotan I, Charlson RW, Ryerson LZ, Levy M and Kister I: Effectiveness of subcutaneous tocilizumab in neuromyelitis optica spectrum disorders. Mult Scler Relat Disord 39: 101920, 2019.

38. Zhang C, Zhang M, Qiu W, Ma H, Zhang X, Zhu Z, Yang CS, Jia D, Zhang TX, Yuan M, et al: Safety and efficacy of tocilizumab versus azathioprine in highly relapsing neuromyelitis optica spectrum disorder (TANGO): An open-label, multicentre, randomised, phase 2 trial. Lancet Neurol 19: 391-401, 2020.

39. Araki M: Blockade of IL-6 signaling in neuromyelitis optica. Neurochem Int 130: 104315, 2019.

40. Yamamura T, Kleiter I, Fujihara K, Palace J, Greenberg B, Zakrzewska-Pniewska B, Patti F, Tsai CP, Saiz A, Yamazaki $\mathrm{H}$, et al: Trial of satralizumab in neuromyelitis optica spectrum disorder. N Engl J Med 381: 2114-2124, 2019.

41. Traboulsee A, Greenberg BM, Bennett JL, Szczechowski L, Fox E, Shkrobot S, Yamamura T, Terada Y, Kawata Y, Wright $\mathrm{P}$, et al: Safety and efficacy of satralizumab monotherapy in neuromyelitis optica spectrum disorder: A randomised, double-blind, multicentre, placebo-controlled phase 3 trial. Lancet Neurol 19: 402-412, 2020.
42. Lotan I, McGowan R and Levy M: Anti-IL-6 therapies for neuromyelitis optica spectrum disorders: A systematic review of safety and efficacy. Curr Neuropharmacol: Apr 28, 2020 (Epub ahead of print). doi: 10.2174/1570159X18666200429010825.

43. Rosso M, Saxena S and Chitnis T: Targeting IL-6 receptor in the treatment of neuromyelitis optica spectrum: A review of emerging treatment options. Expert Rev Neurother 20: 509-516, 2020.

44. Heo YA: Satralizumab: First approval. Drugs 80: 1477-1482, 2020.

45. Carpanini SM, Torvell M and Morgan BP: Therapeutic inhibition of the complement system in diseases of the central nervous system. Front Immunol 10: 362, 2019.

46. Pittock SJ,Lennon VA,McKeon A,MandrekarJ, WeinshenkerBG, Lucchinetti CF, O'Toole O and Wingerchuk DM: Eculizumab in AQP4-IgG-positive relapsing neuromyelitis optica spectrum disorders: An open-label pilot study. Lancet Neurol 12: 554-562, 2013.

47. Pittock SJ, Berthele A, Fujihara K, Kim HJ, Levy M, Palace J, Nakashima I, Terzi M, Totolyan N, Viswanathan S, et al: Eculizumab in aquaporin-4-positive neuromyelitis optica spectrum disorder. N Engl J Med 381: 614-625, 2019.

48. Frampton JE: Eculizumab: A review in neuromyelitis optica spectrum disorder. Drugs 80: 719-727, 2020.

49. Verkman AS, Smith AJ, Phuan PW, Tradtrantip L and Anderson MO: The aquaporin- 4 water channel as a potential drug target in neurological disorders. Expert Opin Ther Targets 21: 1161-1170, 2017.

50. Tradtrantip L, Zhang H, Saadoun S, Phuan PW, Lam C, Papadopoulos MC, Bennett JL and Verkman AS: Antiaquaporin-4 monoclonal antibody blocker therapy for neuromyelitis optica. Ann Neurol 71: 314-322, 2012.

51. Duan T, Tradtrantip L, Phuan PW, Bennett JL and Verkman AS: Affinity-matured 'aquaporumab' anti-aquaporin-4 antibody for therapy of seropositive neuromyelitis optica spectrum disorders. Neuropharmacology 162: 107827, 2020.

52. Shimizu F, Sano Y, Takahashi T, Haruki H, Saito K, Koga M and Kanda T: Sera from neuromyelitis optica patients disrupt the blood-brain barrier. J Neurol Neurosurg Psychiatry 83: 288-297, 2012.

53. Mealy MA, Shin K, John G and Levy M: Bevacizumab is safe in acute relapses of neuromyelitis optica. Clin Exp Neuroimmunol 6: 413-418, 2015.

54. Keating GM: Bevacizumab: A review of its use in advanced cancer. Drugs 74: 1891-1925, 2014.

55. Mealy MA and Levy M: A pilot safety study of ublituximab, a monoclonal antibody against CD20, in acute relapses of neuromyelitis optica spectrum disorder. Medicine (Baltimore) 98: e15944, 2019. 